\title{
STABILITY OF INTERVAL POSITIVE FRACTIONAL DISCRETE-TIME LINEAR SYSTEMS
}

\author{
TADEUSZ KACZOREK ${ }^{a}$
}

\author{
${ }^{a}$ Faculty of Electrical Engineering \\ Białystok Technical University, Wiejska 45D, 15-351 Białystok, Poland \\ e-mail: kaczorek@ee.pw.edu.pl
}

\begin{abstract}
The aim of this work is to show that interval positive fractional discrete-time linear systems are asymptotically stable if and only if the respective lower and upper bound systems are asymptotically stable. The classical Kharitonov theorem is extended to interval positive fractional linear systems.
\end{abstract}

Keywords: interval system, positive system, fractional system, linear discrete-time system, stability.

\section{Introduction}

A dynamical system is called positive if its state variables take nonnegative values for all nonnegative inputs and nonnegative initial conditions. Positive linear systems were investigated by Berman and Plemmons (1994), Farina and Rinaldi (2000) or Kaczorek (2002), along with positive nonlinear systems (Kaczorek, 2016; $2015 \mathrm{~b} ; 2014 ; 2015 \mathrm{a} ; 2015 \mathrm{c})$. Examples of positive systems are industrial processes involving chemical reactors, heat exchangers and distillation columns, storage systems, compartmental systems, water and atmospheric pollution models. A variety of models having positive linear behavior can be found in engineering, management science, economics, social sciences, biology and medicine, etc.

Mathematical fundamentals of fractional calculus are included in the monographs by Ortigueira (2011), Oldham and Spanier (1974) Ostalczyk (2016; 2008) and Podlubny (1999). Fractional dynamical linear and nonlinear systems were investigated by Kaczorek (2016; 2013; 2008; 2018a; 2012b; 2015c), Radwan et al. (2009), Sajewski (2016a; 2016b), Solteiro Pires et al. (2006), Vinagre et al. (2002) or Xiang-Jun et al. (2008).

Positive linear systems with different fractional orders were addressed by Busłowicz (2012), Kaczorek (2010; 2011), Kaczorek and Rogowski (2015), as well as Sajewski, (2016b). Descriptor (singular) linear systems were analyzed by Kaczorek $(2014 ; 2012 b ; 1997)$, along with the stability of a class of nonlinear fractional-order systems (Kaczorek, 2016; 2015c). Application of the Drazin inverse to the analysis of descriptor fractional discrete-time linear systems was presented by Kaczorek (2013). A comparison of three methods of the analysis of descriptor fractional systems was presented by Sajewski (2016a). Stability of linear fractional order systems with delays was analyzed by Busłowicz (2008), and simple conditions for practical stability of positive fractional systems were proposed by Busłowicz and Kaczorek (2009). Stability of interval positive continuous-time linear systems was addressed by Kaczorek (2018b).

In this paper the asymptotic stability of interval positive fractional discrete-time linear systems will be investigated.

The paper is organized as follows. In Section 2 some basic definitions and theorems concerning positivity and stability of fractional discrete-time linear systems are recalled. Stability of interval positive fractional linear systems is analyzed in Section 3. A convex linear combination of Schur polynomials and the stability of interval positive fractional discrete-time linear systems are investigated in Section 4. Concluding remarks are contained in Section 5.

The following notation will be used: $\mathbb{R}$, the set of real numbers; $\mathbb{R}^{n \times m}$, the set of real $n \times m$ matrices; $\mathbb{R}_{+}^{n \times m}$, the set of real $n \times m$ matrices with nonnegative entries and $\mathbb{R}_{+}^{n}=\mathbb{R}_{+}^{n \times 1} ; M_{n}$, the set of $n \times n$ Metzler matrices (real matrices with nonnegative off-diagonal entries); $I_{n}$, the $n \times n$ identity matrix; for $A=\left[a_{i j}\right] \in \mathbb{R}^{n \times n}$ and 
$B=\left[b_{i j}\right] \in \mathbb{R}^{n \times n}$ the inequality $A \geq B$ means $a_{i j} \geq b_{i j}$ for $i, j=1,2, \ldots, n$.

\section{Preliminaries}

Consider the autonomous fractional discrete-time linear system

$$
\Delta^{\alpha} x_{i+1}=A x_{i}, \quad 0<\alpha<1, \quad i \in \mathbb{Z}_{+},
$$

where

$$
\begin{gathered}
\Delta^{\alpha} x_{i}=\sum_{j=1}^{i} c_{j} x_{i-j}, \\
c_{j}=(-1)^{j}\left(\begin{array}{l}
\alpha \\
j
\end{array}\right), \\
\left(\begin{array}{l}
\alpha \\
j
\end{array}\right)= \begin{cases}1 & \text { for } j=0, \\
\frac{\alpha(\alpha-1) \ldots(\alpha-j+1)}{j !} & \text { for } j=1,2, \ldots\end{cases}
\end{gathered}
$$

is the fractional $\alpha$-th order difference of $x_{i}$. Here $x_{i} \in \mathbb{R}^{n}$ and $u_{i} \in \mathbb{R}^{m}$ are the state and input vectors, respectively, and $A \in \mathbb{R}^{n \times n}$.

Substitution of (2) into (1) yields

$$
x_{i+1}=A_{\alpha} x_{i}-\sum_{j=2}^{i+1} c_{j} x_{i-j+1}, \quad i \in \mathbb{Z}_{+},
$$

where

$$
A_{\alpha}=A+I_{n} \alpha \text {. }
$$

Lemma 1. (Kaczorek, 2012a) If $0<\alpha<1$ then

$$
\begin{aligned}
-c_{j} & >0 \text { for } j=1,2, \ldots, \\
\sum_{j=1}^{n} c_{j} & =-1 .
\end{aligned}
$$

Definition 1. (Kaczorek, 2012a) The fractional system (1) is called (internally) positive if $x_{i} \in \mathbb{R}_{+}^{n}, i \in \mathbb{Z}_{+}$for any initial conditions $x_{0} \in \mathbb{R}_{+}^{n}$.

Theorem 1. (Kaczorek, 2012a) The fractional system (1) is positive if and only if

$$
A_{\alpha} \in \mathbb{R}_{+}^{n \times n} .
$$

Definition 2. The fractional positive system (1) is called asymptotically stable if

$$
\lim _{i \rightarrow \infty} x_{i}=0 \quad \text { for all } x_{0} \in \mathbb{R}_{+}^{n} .
$$

Theorem 2. (Kaczorek, 2012a) The fractional positive system (1) is asymptotically stable if and only if one of the following equivalent conditions is satisfied: (i) All coefficients of the characteristic polynomial

$$
\begin{aligned}
p_{A}(z) & =\operatorname{det}\left[I_{n}(z+1)-A\right] \\
& =z^{n}+a_{n-1} z^{n-1}+\cdots+a_{1} z+a_{0}
\end{aligned}
$$

are positive, i.e., $a_{k}>0$, for $k=0,1, \ldots, n-1$.

(ii) All principal minors of the matrix

$$
\begin{aligned}
\bar{A} & =I_{n}-A \\
& =\left[\begin{array}{ccc}
\bar{a}_{11} & \ldots & \bar{a}_{1 n} \\
\vdots & \ldots & \vdots \\
\bar{a}_{n 1} & \ldots & \bar{a}_{n n}
\end{array}\right]
\end{aligned}
$$

are positive, i.e.,

$$
\begin{array}{r}
\left|a_{11}\right|>0, \quad\left|\begin{array}{ll}
\bar{a}_{11} & \bar{a}_{12} \\
\bar{a}_{21} & \bar{a}_{22}
\end{array}\right|>0, \\
\ldots, \quad \operatorname{det} \bar{A}>0 .
\end{array}
$$

(iii) There exists a strictly positive vector $\lambda^{T}=$ $\left[\begin{array}{lll}\lambda_{1} & \cdots & \lambda_{n}\end{array}\right]^{T}, \lambda_{k}>0, k=1, \ldots, n$ such that

$$
\left[A-I_{n}\right] \lambda<0 .
$$

Theorem 3. The fractional positive system (1) with (3b) is asymptotically stable if and only if there exists a strictly positive vector $\lambda>0$ such that

$$
A \lambda<0
$$

Proof. Note that the positive fractional system (3) can be interpreted as a positive linear system with numbers of delays increasing to infinity. It is well known (Kaczorek, 2012a) that the stability of positive discrete-time linear systems depends only on the sum of state matrices

$$
\hat{A}=A_{\alpha}-\sum_{j=2}^{\infty} c_{j} I_{n}
$$

From (4b) we have

$$
-\sum_{j=2}^{\infty} c_{j}=1-\alpha
$$

Substituting (13) into (12), we get

$$
\hat{A}=A_{\alpha}+(1-\alpha) I_{n}=A+I_{n},
$$

since $A_{\alpha}=A+I_{n} \alpha$.

Applying the condition (10) to (14), we obtain (11). 
Example 1. Consider the fractional discrete-time system (1) for $\alpha=0.6$ with the matrix

$$
A=\left[\begin{array}{cc}
-0.4 & 0.2 \\
0.3 & -0.5
\end{array}\right]
$$

The fractional system is positive since the matrix

$$
A_{\alpha}=A+I_{2} \alpha=\left[\begin{array}{cc}
0.2 & 0.2 \\
0.3 & 0.1
\end{array}\right] \in \mathbb{R}_{+}^{2 \times 2}
$$

has positive entries. It is asymptotically stable since for $\lambda^{T}=\left[\begin{array}{ll}1 & 1\end{array}\right]$ we have

$$
A \lambda=\left[\begin{array}{cc}
-0.4 & 0.2 \\
0.3 & -0.5
\end{array}\right]\left[\begin{array}{l}
1 \\
1
\end{array}\right]=\left[\begin{array}{l}
-0.2 \\
-0.2
\end{array}\right]<0
$$

and the condition (11) is satisfied.

Consider the set (family) of the $n$-th degree polynomials

$$
p_{n}(s):=a_{n} s^{n}+a_{n-1} s^{n-1}+\cdots+a_{1} s+a_{0}
$$

with interval coefficients

$$
\underline{a_{i}} \leq a_{i} \leq \overline{a_{i}}, \quad i=0,1, \ldots, n .
$$

Using (18a), we define the following four polynomials:

$$
\begin{aligned}
p_{1 n}(s):= & \underline{a_{0}}+\underline{a_{1}} s+\overline{a_{2}} s^{2}+\overline{a_{3}} s^{3} \\
& +\underline{a_{4}} s^{4}+\underline{a_{5}} s^{5}+\ldots, \\
p_{2 n}(s):= & \underline{a_{0}}+\overline{a_{1}} s+\overline{a_{2}} s^{2}+\underline{a_{3}} s^{3} \\
& +\underline{a_{4}} s^{4}+\overline{a_{5}} s^{5}+\ldots, \\
p_{3 n}(s):= & \overline{a_{0}}+\underline{a_{1}} s+\underline{a_{2}} s^{2}+\overline{a_{3}} s^{3} \\
& +\overline{a_{4}} s^{4}+\underline{a_{5}} s^{5}+\ldots, \\
p_{4 n}(s):= & \overline{a_{0}}+\overline{a_{1}} s+\underline{a_{2}} s^{2}+\underline{a_{3}} s^{3} \\
& +\overline{a_{4}} s^{4}+\overline{a_{5}} s^{5}+\ldots
\end{aligned}
$$

Theorem 4. (Kharitonov, 1978) The set of polynomials (18) is asymptotically stable if and only if the four polynomials (19) are asymptotically stable.

A proof of this result, also called the Kharitonov theorem, can be found in the work of Kaczorek (2012a).

\section{Fractional interval positive linear continuous-time systems}

Consider the interval fractional positive discrete-time linear system (1) with the interval matrix $A \in \mathbb{R}_{+}^{n \times n}$ defined by

$$
A_{1} \leq A \leq A_{2}
$$

or, equivalently,

$$
A \in\left[A_{1}, A_{2}\right]
$$

Definition 3. The interval fractional positive system with (20) is called asymptotically stable if the system is asymptotically stable for all matrices $A \in \mathbb{R}_{+}^{n \times n}$ belonging to the interval $\left[A_{1}, A_{2}\right]$.

Definition 4. The matrix

$$
\begin{aligned}
A=(1-k) A_{1}+k A_{2}, \quad 0 \leq k \leq 1, & \\
A_{1} & \in \mathbb{R}^{n \times n}, \quad A_{2} \in \mathbb{R}^{n \times n}
\end{aligned}
$$

is called the convex linear combination of matrices $A_{1}$ and $A_{2}$.

Theorem 5. The convex linear combination (21) is asymptotically stable if and only if the matrices $A_{1} \in$ $\mathbb{R}^{n \times n}$ and $A_{2} \in \mathbb{R}^{n \times n}$ are asymptotically stable.

Proof. If the matrices $A_{1} \in \mathbb{R}^{n \times n}$ and $A_{2} \in \mathbb{R}^{n \times n}$ are asymptotically stable then by 111 there exists a strictly positive vector $\lambda \in \mathbb{R}_{+}^{n}$ such that

$$
A_{l} \lambda<0 \quad \text { for } l=1,2 .
$$

In this case, using (21) and (22), we obtain

$$
\begin{aligned}
A \lambda & =\left[(1-k) A_{1}+k A_{2}\right] \lambda \\
& =(1-k) A_{1} \lambda+k A_{2} \lambda<0 \quad \text { for } 0 \leq k \leq 1 .
\end{aligned}
$$

Therefore, if the matrices $A_{l}, l=1,2$ are asymptotically stable, then the convex linear combination (21) is also asymptotically stable. The necessity follows immediately from the fact that $k$ can be equal to zero and one.

Theorem 6. The interval fractional positive system (1) with (20) is asymptotically stable if and only if the matrices $A_{1} \in \mathbb{R}^{n \times n}$ and $A_{2} \in \mathbb{R}^{n \times n}$ are Schur matrices.

Proof. By (11) the matrices $A_{1} \in \mathbb{R}^{n \times n}$ and $A_{2} \in \mathbb{R}^{n \times n}$ are Schur matrices if and only if there exists a strictly positive vector $\lambda \in \mathbb{R}_{+}^{n}$ such that (22) holds. The convex linear combination (21) satisfies the condition $A \lambda<0$ if and only if 22 holds. Therefore, the interval fractional positive systems (10) with (20) is asymptotically stable if and only if $A_{1} \in \mathbb{R}^{n \times n}$ and $A_{2} \in \mathbb{R}^{n \times n}$ are Schur matrices.

Example 2. Consider the interval fractional positive linear systems (1) with the matrices

$$
A_{1}=\left[\begin{array}{cc}
-0.3 & 0.1 \\
0.05 & -0.4
\end{array}\right], \quad A_{2}=\left[\begin{array}{cc}
-0.5 & 0.3 \\
0.2 & -0.6
\end{array}\right]
$$

It is easy to check that for $\lambda^{T}=\left[\begin{array}{ll}1 & 1\end{array}\right]$ we have

$$
\begin{aligned}
& A_{1} \lambda=\left[\begin{array}{cc}
-0.3 & 0.1 \\
0.05 & -0.4
\end{array}\right]\left[\begin{array}{l}
1 \\
1
\end{array}\right]=\left[\begin{array}{c}
-0.2 \\
-0.35
\end{array}\right]<0 \\
& A_{2} \lambda=\left[\begin{array}{cc}
-0.5 & 0.3 \\
0.2 & -0.6
\end{array}\right]\left[\begin{array}{l}
1 \\
1
\end{array}\right]=\left[\begin{array}{l}
-0.2 \\
-0.4
\end{array}\right]<0
\end{aligned}
$$


Therefore, by Theorem 5 the interval fractional positive system (1) with (20) is asymptotically stable.

\section{Convex linear combination of Schur polynomials and stability of interval fractional positive linear systems}

Definition 5. The polynomial

$$
p(z)=b_{n} z^{n}+b_{n-1} z^{n-1}+\cdots+b_{1} z+b_{0}
$$

is called a Schur polynomial if its zeros $z_{l}, l=1, \ldots, n$ satisfy the condition

$$
\left|z_{l}\right|<1 \quad \text { for } l=1, \ldots, n .
$$

Definition 6. The polynomial

$$
p(z)=(1-k) p_{1}(z)+k p_{2}(z)
$$

for $k \in[0,1]$ is called a convex linear combination of the polynomials

$$
\begin{aligned}
p_{i}(z)= & b_{i, n} z^{n}+b_{i, n-1} z^{n-1}+\ldots \\
& +b_{i, 1} z+b_{i, 0}, \quad i=1,2 .
\end{aligned}
$$

Theorem 7. (Kaczorek, 2018b) Any convex linear combination of Hurwitz polynomials is also a Hurwitz polynomial.

For positive linear systems we have the following relationship between Hurwitz and Schur polynomials.

Theorem 8. The polynomial

$$
p(s)=a_{n} s^{n}+a_{n-1} s^{n-1}+\cdots+a_{1} s+a_{0}
$$

is Hurwitz and the polynomial

$$
p(z)=b_{n} z^{n}+b_{n-1} z^{n-1}+\cdots+b_{1} z+b_{0}
$$

is a Schur polynomial if and only if their coefficients $a_{i}$ and $b_{i} i=0,1, \ldots, n$ are related by

$$
\begin{aligned}
a_{0} & =b_{0}+b_{1}+\cdots+b_{n}, \\
a_{1} & =b_{1}+2 b_{2}+\cdots+n b_{n}, \\
& \vdots \\
a_{n-1} & =b_{n-1}+n b_{n}, \\
a_{n} & =b_{n} .
\end{aligned}
$$

Proof. It is well known (Kaczorek, 2012a) that for positive linear discrete-time and continuous-time systems the zeros $z_{l}, l=1, \ldots, n$ of the polynomial (31) and the zeros $s_{l}, l=1, \ldots, n$ of the polynomial (30) are related by

$$
z_{l}=s_{l}+1, \quad l=1, \ldots, n .
$$

Substituting $z=s+1$ into the polynomial (31), we obtain

$$
\begin{array}{r}
b_{n}(s+1)^{n}+b_{n-1}(s+1)^{n-1}+\cdots+b_{1}(s+1)+b_{0} \\
=a_{n} s^{n}+a_{n-1} s^{n-1}+\cdots+a_{1} s+a_{0}
\end{array}
$$

and it is easy to verify that the coefficients $a_{i}$ and $b_{i} i=$ $0,1, \ldots, n$ are related by (32).

The polynomial (30) is Hurwitz if and only if $a_{i}>0$ for $i=0,1, \ldots, n$ and the polynomial (31) is Schur if and only if $b_{i}>0$ for $i=0,1, \ldots, n$. From (32) it follows that $b_{i}>0, i=0,1, \ldots, n$ implies $a_{i}>0$ for $i=0,1, \ldots, n$.

Example 3. The polynomial

$$
p(z)=z^{2}+0.6 z+0.08
$$

of a positive discrete-time linear system is a Schur polynomial since its zeros are $z_{1}=-0.2$ and $z_{2}=-0.4$.

Substituting $z=s+1$ into (35), we obtain

$$
\begin{aligned}
p(s) & =(s+1)^{2}+0.6(s+1)+0.08 \\
& =s^{2}+2.6 s+1.68
\end{aligned}
$$

with zeros $s_{1}=-1.2$ and $s_{2}=-1.4$. Therefore, the polynomial (36) is Hurwitz.

Theorem 9. The interval positive fractional discretetime linear system with the characteristic polynomial 31. with interval coefficients $\underline{b}_{i} \leq b_{i} \leq \bar{b}_{i}$ is asymptotically stable if and only if the lower bounds $\underline{b}_{i}, i=0,1, \ldots, n$ are positive.

Proof. Observe that from (32) it follows that $\underline{b}_{i}>0$, $i=0,1, \ldots, n$ implies $a_{i}>0$ for $i=0,1, \ldots, n$ and the characteristic polynomial (30) is Hurwitz. By Theorem 2 the continuous-time system is asymptotically stable. A similar result is obtained for the upper bound. Therefore, the interval fractional positive discrete-time system (31) is asymptotically stable if the lower and upper bounds of the coefficients are positive.

Remark 1. The equalities (32) can be used to compute the lower and upper bounds of the coefficients $a_{i}, i=$ $0,1, \ldots, n$ of polynomial 30 knowing the lower and upper bounds of the coefficients $b_{i}, i=0,1, \ldots, n$ of polynomial 31.

Example 4. Consider the characteristic polynomial

$$
p(z)=b_{2} z^{2}+b_{1} z+b_{0}
$$

of positive fractional discrete-time systems with the interval coefficients

$$
1 \leq b_{2} \leq 3, \quad 2 \leq b_{1} \leq 3, \quad 1 \leq b_{0} \leq 4
$$


The equivalent characteristic polynomial of the continuous-time system has the form

$$
\begin{aligned}
p(s) & =b_{2}(s+1)^{2}+b_{1}(s+1)+b_{0} \\
& =a_{2} s^{2}+a_{1} s+a_{0},
\end{aligned}
$$

where

$$
a_{2}=b_{2}, \quad a_{1}=b_{1}+2 b_{2}, \quad a_{0}=b_{0}+b_{1}+b_{2} .
$$

Therefore, the interval coefficients of the characteristic polynomial of the continuous-time system are

$$
1 \leq a_{2} \leq 3, \quad 4 \leq a_{1} \leq 9, \quad 4 \leq a_{0} \leq 10 .
$$

By Theorem 9 the interval positive discrete-time linear system with the characteristic polynomial 37 is asymptotically stable since the lower and upper bounds 41) are positive.

\section{Concluding remarks}

The asymptotic stability of interval fractional positive linear discrete-time systems has been investigated. It has been shown that:

1. The interval fractional positive system (1) with (20) is asymptotically stable if and only if the matrices $A_{i}, \quad i=1,2$, are Schur matrices (Theorem 6).

2. Any convex linear combination of Hurwitz polynomials is also a Hurwitz polynomial (Theorem 7).

3. An interval fractional positive system is asymptotically stable if the lower and upper bounds to the coefficients of its characteristic polynomial are positive (Theorem 9).

The discussion has been illustrated with numerical examples of positive interval discrete-time systems. An open problem is an extension to continuous-time and discrete-time standard (nonpositive) fractional linear systems.

\section{Acknowledgment}

The research has been carried out in the framework of the project no. S/WE/1/2016 and financed with the funds for science by the Polish Ministry of Science and Higher Education.

\section{References}

Berman, A. and Plemmons R.J. (1994). Nonnegative Matrices in the Mathematical Sciences, SIAM, Philadelphia, PA.
Busłowicz, M. (2008). Stability of linear continuous-time fractional order systems with delays of the retarded type, Bulletin of the Polish Academy of Sciences: Technical Sciences 56(4): 319-324.

Busłowicz, M. (2012). Stability analysis of continuous-time linear systems consisting of $n$ subsystems with different fractional orders, Bulletin of the Polish Academy of Sciences: Technical Sciences 60(2): 279-284.

Busłowicz, M. and Kaczorek, T. (2009). Simple conditions for practical stability of positive fractional discrete-time linear systems, International Journal of Applied Mathematics and Computer Science 19(2): 263-269, DOI: 10.2478/v10006-009-0022-6.

Farina, L. and Rinaldi, S. (2000). Positive Linear Systems: Theory and Applications, Wiley, New York, NY.

Kaczorek, T. (1997). Positive singular discrete-time linear systems, Bulletin of the Polish Academy of Sciences: Technical Sciences 45(4): 619-631.

Kaczorek, T. (2002). Positive $1 D$ and $2 D$ Systems, Springer, London.

Kaczorek, T. (2008). Fractional positive continuous-time linear systems and their reachability, International Journal of Applied Mathematics and Computer Science 18(2): 223-228, DOI: 10.2478/v10006-008-0020-0.

Kaczorek, T. (2010). Positive linear systems with different fractional orders, Bulletin of the Polish Academy of Sciences: Technical Sciences 58(3): 453-458.

Kaczorek, T. (2011). Positive linear systems consisting of $n$ subsystems with different fractional orders, IEEE Transactions on Circuits and Systems 58(7): 1203-1210.

Kaczorek, T. (2012a). Selected Problems of Fractional Systems Theory, Springer, Berlin.

Kaczorek, T. (2012b). Positive fractional continuous-time linear systems with singular pencils, Bulletin of the Polish Academy of Sciences: Technical Sciences 60(1): 9-12.

Kaczorek, T. (2013). Application of the Drazin inverse to the analysis of descriptor fractional discrete-time linear systems with regular pencils, International Journal of Applied Mathematics and Computer Science 23(1): 29-33, DOI: 10.2478/amcs-2013-0003.

Kaczorek, T. (2014). Descriptor positive discrete-time and continuous-time nonlinear systems, in R.S. Romaniuk (Ed.), Photonics Applications in Astronomy, Communications, Industry, and High-Energy Physics Experiments, Proceedings of SPIE, Vol. 9290, SPIE, Cardiff, pp. 1-11.

Kaczorek, T. (2015a). Positivity and stability of discrete-time nonlinear systems, IEEE 2nd International Conference on Cybernetics, Gdynia, Poland, pp. 156-159.

Kaczorek, T. (2015b). Analysis of positivity and stability of discrete-time and continuous-time nonlinear systems, Computational Problems of Electrical Engineering 5(1): 11-16.

Kaczorek, T. (2015c). Stability of fractional positive nonlinear systems, Archives of Control Sciences 25(4): 491-496. 
Kaczorek, T. (2016). Analysis of positivity and stability of fractional discrete-time nonlinear systems, Bulletin of the Polish Academy of Sciences: Technical Sciences 64(3): 491-494.

Kaczorek, T. (2018a). Positivity and stability of standard and fractional descriptor continuous-time linear and nonlinear systems, International Journal of Nonlinear Sciences and Numerical Simulation, (in press).

Kaczorek, T. (2018b). Stability of interval positive continuous-time linear systems, Bulletin of the Polish Academy of Sciences: Technical Sciences 66(1): 31-35.

Kaczorek, T. and Rogowski, K. (2015). Fractional Linear Systems and Electrical Circuits, Springer, Cham.

Kharitonov, V.L. (1978). Asymptotic stability of an equilibrium position of a family of systems of differential equations, Differentsial'nye uravneniya 14(11): 2086-2088.

Ortigueira, M. D. (2011). Fractional Calculus for Scientists and Engineers, Springer, Dordrecht.

Oldham, K.B. and Spanier, J. (1974). The Fractional Calculus, Academic Press, New York, NY.

Ostalczyk, P. (2008). Epitome of the Fractional Calculus: Theory and Its Applications in Automatics, Publishing House of the Łódź University of Technology, Łódź, (in Polish).

Ostalczyk, P. (2016). Discrete Fractional Calculus, World Scientific, River Edge, NJ.

Podlubny, I. (1999). Fractional Differential Equations, Academic Press, San Diego, CA.

Radwan, A.G., Soliman, A.M., Elwakil, A.S. and Sedeek A. (2009). On the stability of linear systems with fractional-order elements. Chaos, Solitones and Fractals 40(5): 2317-2328.

Sajewski, Ł (2016a). Descriptor fractional discrete-time linear system and its solution-comparison of three different methods, in R. Szewczyk et al. (Eds.), Challenges in Automation, Robotics and Measurement Techniques, Springer, Cham, pp. 37-50.
Sajewski, Ł. (2016b). Descriptor fractional discrete-time linear system with two different fractional orders and its solution, Bulletin of the Polish Academy of Sciences: Technical Sciences 64(1): 15-20.

Solteiro Pires, E.J., Tenreiro Machado, J.A. and de Moura Oliveira, P.B. (2006). Fractional dynamics in genetic algorithms, IFAC Proceedings Volumes 39(11): 414-419.

Vinagre, B.M., Monje, C.A. and Calderon A.J. (2002) Fractional order systems and fractional order control actions, Proceedings of the Conference on Decision and Control, Las Vegas, NV, USA, pp. 2550-2554.

Xiang-Jun, W., Zheng-Mao, W. and Jun-Guo, L. (2008) Stability analysis of a class of nonlinear fractional-order systems, IEEE Transactions on Circuits and Systems II: Express Briefs 55(11): 1178-1182.

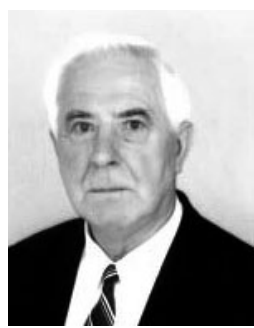

Tadeusz Kaczorek received his $\mathrm{MSc}, \mathrm{PhD}$ and DSc degrees in electrical engineering from the Warsaw University of Technology in 1956, 1962 and 1964, respectively. In the years 1968-1969 he was the dean of the Electrical Engineering Faculty, and in the period of 1970-1973 he was a deputy rector of the Warsaw University of Technology. In 1971 he became a professor and in 1974 a full professor at the same university. Since 2003 he has been a professor at the Białystok University of Technology. In 1986 he was elected a corresponding member and in 1996 a full member of the Polish Academy of Sciences. In the years 1988-1991 he was the director of the Research Center of the Polish Academy of Sciences in Rome. In 2004 he was elected an honorary member of the Hungarian Academy of Sciences. He has been granted honorary doctorates by 13 universities. His research interests cover systems theory, especially singular multidimensional systems, positive multidimensional systems, singular positive 1D and 2D systems, as well as positive fractional $1 \mathrm{D}$ and $2 \mathrm{D}$ systems. He initiated research in the field of singular 2D, positive 2D and positive fractional linear systems. $\mathrm{He}$ has published 28 books ( 8 in English) and over 1100 scientific papers. $\mathrm{He}$ has also supervised $69 \mathrm{PhD}$ theses. He is the editor-in-chief of the Bulletin of the Polish Academy of Sciences: Technical Sciences and a member of editorial boards of ten international journals.

Received: 13 August 2017 Revised: 6 December 2017

Accepted: 4 March 2018 